\title{
Dynamical Models for Disk Galaxies with Triaxial Halos
}

\author{
Lawrence M. Widrow* \\ Department of Physics, Engineering Physics, and Astronomy, Queen's University, \\ Kingston, ON, KrL 3N6, Canada \\ (submitted to the Astrophysical Journal, October, 200\%)
}

\begin{abstract}
We construct self-consistent dynamical models for disk galaxies with triaxial, cuspy halos. We begin with an equilibrium, axisymmetric, disk-bulge-halo system and apply an artificial acceleration to the halo particles. By design, this acceleration conserves energy and thereby preserving the system's differential energy distribution even as its phase space distribution function is altered. The halo becomes triaxial but its spherically-averaged density profile remains largely unchanged. The final system is in equilibrium, to a very good approximation, so long as the halo's shape changes adiabatically. The disk and bulge are "live" while the halo is being deformed; they respond to the changing gravitational potential but also influence the deformation of the halo. We test the hypothesis that halo triaxiality can explain the rotation curves of low surface brightness galaxies by modelling the galaxy F568-3.
\end{abstract}

Subject headings: Galaxy: kinematics and dynamics — methods: statistical methods: N-body simulations — cosmology: dark matter

\section{INTRODUCTION}

Dark matter halos - at least the ones found in cosmological simulations - have a number of universal traits. Most famously, their density profiles have a shape that is nearly independent of mass, formation epoch, and cosmological model (Navarro. Frenk \& White 1996). Their angular momentum distribution (Bullock et al. 2001), phase space density (Taylor \& Navarro 2001), and velocity anisotropy (Hansen \& Moore 2006) profiles also appear to follow universal forms. In other respects, halos are rather diverse. Simulated halos

\footnotetext{
1widrow@astro.queensu.ca
} 
are typically triaxial with axis ratios that range from 0.6 to 1 (See Dubinski \& Carlberg (1991); Warren et al. (1992) and more recently, Novak et al. (2006)). The shapes of real halos are more difficult to determine but promising observational approaches do exist. Probes of the Galactic halo include flaring of the gas disk (Olling \& Merrifield 2000) and tidal streams of satellite galaxies (Johnston et al. 1999). The shapes of halos in other galaxies can be determined, at least statistically, by weak gravitational lensing surveys (Hoekstra, Yee, \& Gladders 2004; Mandelbaum et al. 2006; Parker et al. 2007). On the other hand, triaxiality can bias attempts to determine a halo's density profile from the rotation curve of the disk that sits within it (Hayashi \& Navarro 2006).

Our main goal in writing this paper is to introduce a novel scheme to generate selfconsistent dynamical models for disk galaxies with triaxial halos. Our models can be tailored to fit observational data for specific galaxies and therefore provide a testing ground to study the disk-halo connection. We consider the effects of halo triaxiality on the rotation curves of low surface brightness galaxies (LSBs) and briefly discuss other applications of the models.

Halos in simulations of a cold dark matter (CDM) universe have central cusps with $\rho \propto r^{-\gamma}$ where $\gamma \simeq 1$ (Navarro, Frenk \& White 1996). In dark matter-dominated galaxies, this density profile would seem to imply a rotation curve where $v \propto r^{1 / 2}$ as $r \rightarrow 0$. By contrast, a halo with a constant density core implies $v \propto r$ as $r \rightarrow 0$. LSBs, which are believed to be dark-matter dominated at small radii, have rotation curves that generally favor a constant density core over a $\gamma=-1$ cusp. This result represents one of the most serious challenges to the CDM scenario (Moore 1994; Flores \& Primack 1994; McGaugh \& de Blok 1998) and has inspired some rather exotic alternatives. De Blok \& McGaugh (1998), for example, suggested that LSB rotation curves could be explained by Modified Newtonian Gravity while Firmani (2000) and Mo \& Mao (2000)) invoked dark matter self-interactions to flatten the central cusp of the halo.

The connection described above between a galaxy's rotation curve and the intrinsic density profile of its halo assumes that the halo is spherically symmetric. However, if a galaxy's halo is triaxial, then gas in the disk will move on non-circular orbits and, under certain conditions, the observed rotation curve will rise approximately linearly even if the intrinsic halo density profile has a steep cusp. Hayashi \& Navarro (2006) and Hayashi, Navarro, \& Springel (2007) presented this argument as a means of reconciling LSB rotation curves with the predictions of the CDM model.

Havashi \& Navarro (2006) and Havashi. Navarro, \& Springel (2007) derived model rotation curves by calculating closed orbits in the potential generated by a triaxial halo. In this paper, we derive rotation curves by making pseudo-observations of a disk that is embedded in the halo. Deviations from axial symmetry in disk and halo are generated concurrently 
and self-consistently.

A number of methods exist for constructing models of, and embedding disks in, triaxial halos. For example, Moore et al. (2004) show that the remnant of a major merger between two equilibrium spherical halos is triaxial. Bailin et al. (2007) describe how to set up an equilibrium disk in a combined halo-disk potential. Our approach produces an Nbody galaxy complete with disk, bulge, and triaxial halo. (Central black holes may also be included, as in Widrow \& Dubinski (2005).) It is inspired by the method outlined in Holley-Bockelmann et al. (2001). In that scheme, dubbed "adiabatic squeezing", particles of an equilibrium halo are subjected to an artificial drag by modifying the force law of a standard N-body code and evolving the system forward in time. A triaxial halo is created if the drag has a different strength along three orthogonal directions, that is, along what become the three principle axes of the halo. The final model will be in equilibrium, to a good approximation, so long as the timescale for the halo's shape to change is slow as compared to the typical orbital timescale of the system.

Adiabatic squeezing causes a halo to shrink in size. For an isolated halo, this shrinking can be reversed by simply rescaling the positions and velocities of the particles. Obviously, this method is unsuitable for disk-bulge-halo systems since the disk and bulge would be disrupted in an unphysical way. We propose a modification of this method in which drag is applied along one axis and "negative drag" is applied along the other two or vice versa depending on whether one wants a prolate or oblate halo. We require that for each particle, the change in energy due to the artificial drag force is zero. In this way, we change the phase space distribution of the particles but not their energy distribution. As noted in Binney \& Tremaine (1987), if two systems have the same energy distribution, their spherically-averaged density profiles will be very similar even if their phase space distribution functions are different.

Our starting point is the equilibrium model of Widrow, Pym, \& Dubinski (2007) which comprises a Sersic bulge, cuspy dark halo, and exponential disk. The model is described in terms of a phase space distribution function (DF) which, in turn, is a function of the integrals of motion. In the current version of the model, the halo component of the DF depends only on the energy. In the absence of a disk, the halo is spherically symmetric. With the disk included, the halo is flattened slightly but is still axisymmetric. Adiabatic deformation allows us to extend our disk-bulge-halo model to systems with triaxial halos.

In Section 2, we describe our method and construct an example of an isolated triaxial halo. We then consider the LSB galaxy F568-3. In Section 3, we present axisymmetric, equilibrium models for this galaxy based on its published surface brightness profile and circular speed curve. In Section 4, we show how transforming the axisymmetric halo in one 
of these models into a triaxial halo changes the shape of the rotation curve. We conclude in Section 5, by summarizing our results and briefly discussing further applications of the method.

\section{METHOD}

We begin with an N-body equilibrium halo and evolve the system forward in time using standard techniques augmented by an artificial, energy-conserving acceleration. To be precise, we introduce an acceleration into the equations of motion given by

$$
\begin{aligned}
& a_{x}=\frac{\left(\beta_{1}-\beta_{2}\right) v_{y}^{2}+\beta_{1} v_{z}^{2}}{v^{2}} v_{x}, \\
& a_{y}=\frac{\left(\beta_{2}-\beta_{1}\right) v_{x}^{2}+\beta_{2} v_{z}^{2}}{v^{2}} v_{y},
\end{aligned}
$$

and

$$
a_{z}=-\frac{\beta_{1} v_{x}^{2}+\beta_{2} v_{y}^{2}}{v^{2}} v_{z}
$$

where $v$ is the speed of the particle and $\mathbf{a} \cdot \mathbf{v}=0$, as required. The coefficients $\beta_{1}$ and $\beta_{2}$ are time-dependent. Following Holley-Bockelmann et al. (2001) we assume that $\beta_{1}$ and $\beta_{2}$ "turn on" at $t=0$ and increase to their respective maximum values over a period $T_{G}$ with a time-dependence given by

$$
\beta_{i}=\beta_{i, \max }\left(3\left(t / T_{G}\right)^{2}-2\left(t / T_{G}\right)^{3}\right) .
$$

$\beta_{1}$ and $\beta_{2}$ remain constant for a time $T_{C}$ before decreasing to zero over a time $T_{D}$.

A few comments regarding the parameters $\beta_{1}, \beta_{2}, T_{G}, T_{C}$, and $T_{D}$ are in order. First, the degree by which the halo departs from spherical symmetry is given, roughly, by the integral $\int \beta_{i} d t=\beta_{i}\left(T_{G}+T_{C}+T_{D}\right)$. Rescaling the $\beta$ 's by a factor $f$ and the $T$ 's by a factor $f^{-1}$ leaves the final halo shape unchanged with one important caveat. $\beta^{-1}$ sets the timescale over which the halo's shape changes and therefore must be longer than its dynamical time $\sim a_{h} / \sigma_{h}$ in order to maintain adiabaticity.

In the case of an isolate halo equations 103 admit several discrete symmetries. For example, interchanging $\beta_{1}$ and $\beta_{2}$ is equivalent to interchanging $x$ and $y$. Similar symmetries are listed in Table 1 . The presence of a disk breaks these symmetries. In general, increasing $\beta_{1}$ causes the system to expand along the $x$-axis while increasing $\beta_{2}$ causes the system to expand along the $y$-axis. Models with $\beta_{1}=\beta_{2}$ are axisymmetric about the $z$-axis. 
As an illustration, we transform an isolated, spherically-symmetric halo into one that is triaxial. We begin with a halo whose density profile is given by

$$
\tilde{\rho}_{\text {halo }}=\frac{2^{2-\gamma} \sigma_{h}^{2}}{4 \pi a_{h}^{2}} \frac{1}{\left(r / a_{h}\right)^{\gamma}\left(1+r / a_{h}\right)^{3-\gamma}} \operatorname{erfc}\left(\frac{r-r_{h}}{\sqrt{2} \delta r_{h}}\right) .
$$

For this example, we set $\gamma=1$ (the NFW value), $a_{h}=10 \mathrm{kpc}, \sigma_{h}=100 \mathrm{~km} \mathrm{~s}^{-1}, r_{h}=100 \mathrm{kpc}$ and $\delta r_{h}=10 \mathrm{kpc}$. We choose $\beta_{1, \max }=0.24 \mathrm{Gyr}^{-1}, \beta_{2, \max }=0.08 \mathrm{Gyr}^{-1}, T_{G}=T_{D}=1 \mathrm{Gyr}$ and $t_{c}=3 \mathrm{Gyr}$. As required, the characteristic timescale for the halo, $a_{h} / \sigma_{h}=100 \mathrm{Myr}$, is short compared to the timescale, $\beta^{-1}=4-12 \mathrm{Gyr}$, associated with the artificial force. Our choice for the $\beta_{i}$ yields a halo whose short axis is along the $z$-direction and whose long axis is along the $x$-direction.

The model is evolved forward in time using the N-body code from Stiff (2003) which is based on the algorithm described in Dehnen (2000). The code uses a multipole expansion for cell-cell interactions; computational costs scale approximately linearly with particle number $N$. The softening length is $200 \mathrm{pc}$ and the timestep is $1 \mathrm{Myr}$. The system is evolved for a period of 15 Gyr.

In Figure 1, we show a contour plot of the projected surface density of the halo along the three principle axes. Note that the departure from spherical symmetry is strongest in the inner parts of the halo. In Figure 2, we show the axis ratios as a function of time. To be precise, we model the density field as an ellipsoidal distribution,

$$
\rho=\rho(\tilde{r}) \quad \text { where } \quad \tilde{r}=x^{2}+\frac{y^{2}}{b^{2}}+\frac{z^{2}}{c^{2}} .
$$

The parameters $b$ and $c$ are calculated through an iterative procedure as outlined in Dubinski \& Carlberg (1991). We show the results using the inner third of the particles, the inner two thirds of the particles, and all of the particles. Again, we see that the halo is more spherical in the outer parts. Note that the axis ratios at all radii oscillate a bit at $t=5 \mathrm{Gyr}$, the time when the artificial acceleration is turned off. After this time, the axis ratios in the inner two thirds of the halo quickly settle down to constant values. The oscillations damp more slowly in the outer parts of the halo where the dynamical time is not much shorter than $\beta_{i}^{-1}$. One can minimize the oscillations by increasing the $T^{\prime} s$ and decreasing the $\beta$ 's but at the cost of additional computation time.

In Figure 3, we show the spherically-averaged differential mass profile, $d M / d r \propto r^{2} \rho$ for the initial model and for the deformed model at $t=6 \mathrm{Gyr}$ and $t=12 \mathrm{Gyr}$ and compare with equation 5. Also shown is the density profile calculated from the initial conditions and the density profile for the system evolved to $12 \mathrm{Gyr}$ with no artificial acceleration. The former illustrates the role mass resolution plays on the measured density profile while the 
latter illustrates the effects of force softening and two-body relaxation. We see that the spherically-averaged density profile is preserved to within the fluctuations introduced by these other effects.

Table 2 presents results for the axes ratios for other choices of $\beta_{1}$ and $\beta_{2}$. Note that models 1a-c (and likewise models $2 \mathrm{a}-\mathrm{b}$ and models $3 \mathrm{a}-\mathrm{c}$ ) are equivalent through the symmetries described in Table 1.

\section{THE LSB GALAXY F568-3}

In this section, we construct axisymmetric, equilibrium models for F568-3, an LSB galaxy which has appeared in a number of studies. We describe our general axisymmetric disk-bulge-halo models, review published photometric and kinematic observations for this galaxy, and discuss the statistical techniques used to tailor the model to the data.

\subsection{Equilibrium Models for Disk-Bulge-Halo Systems}

Our starting point is the dynamical galactic model described in Widrow, Pym, \& Dubinski (2007). The model is axisymmetric and comprises an exponential disk, a Sersic bulge, and a halo whose density profile is given by equation 5. DFs for the bulge and halo are functions of the energy, E, and constructed via an Abel integral transform. The DF for the disk is constructed from three integrals of motion following the method outlined in Kuijken \& Dubinski (1995). The total DF for the composite system self-consistently satisfies the collisionless Boltzmann and Poisson equations.

The bulge has a spherically-averaged density profile given, to a good approximation, by

$$
\tilde{\rho}_{\text {bulge }}(r)=\rho_{b}\left(\frac{r}{R_{e}}\right)^{-p} e^{-b\left(r / R_{e}\right)^{1 / n}} .
$$

This density profile yields the Sersic law,

$$
\Sigma(r)=\Sigma_{0} e^{-b\left(R / R_{e}\right)^{1 / n}}
$$

for the projected mass density provided one sets $p=1-0.6097 / n+0.05563 / n^{2}$ Prugniel \& Simien 1997; Terzić \& Graham 2005). $\Sigma_{0}, R_{e}$ and $n$ are free parameters while the constant $b$ is adjusted so that $R_{e}$ encloses half the total projected light or mass.

The disk DF depends on $E$, the angular momentum about the symmetry axis, $L_{z}$, and an approximate integral of motion, $E_{z}$, which corresponds to the energy associated 
with vertical motions of stars in the disk. The DF is adjusted so that the intrinsic threedimensional density distribution and velocity dispersion profile are given, respectively, by

$$
\rho_{\text {disk }}(R, z)=\rho_{0} \exp ^{-R / R_{d}} \operatorname{sech}^{2}\left(z / z_{d}\right) \operatorname{erfc}\left(\left(R-R_{\text {out }}\right) / \delta R_{\text {out }}\right)
$$

and

$$
\sigma_{R}^{2}(R)=\sigma_{R 0}^{2} \exp \left(-R / R_{\sigma}\right)
$$

\section{2. $\quad$ Surface Brightness Profile and Rotation Curve for F568-3}

Multi-band photometry for the LSB galaxy F568-3 is presented in de Blok, van der Hulst, \& Bothun (1995). The galaxy resembles a normal late-type galaxy exhibiting a disk and faint spiral arms. However, its central B-band surface brightness is more than a magnitude fainter than the Freeman value (Freeman 1970) placing it squarely in the category of LSBs. For the purpose of modelling the galaxy, we use the R-band surface brightness profile from Figure 2 of (de Blok, van der Hulst, \& Bothun 1995).

High-resolution rotation curves for F568-3 are described in McGaugh, Rubin, \& de Blok (2001). The circular speed rises approximately linearly to $80 \mathrm{~km} \mathrm{~s}^{-1}$ within $4 \mathrm{kpc}$. It continues to rise beyond this radius reaching a maximum value of $\sim 100 \mathrm{~km} \mathrm{~s}^{-1}$ at $R \simeq 12 \mathrm{kpc}$.

\subsection{Markov Chain Monte Carlo Analysis of F568-3}

We use Bayesian statistics and the Markov Chain Monte Carlo (MCMC) method to find suitable axisymmetric models for F568-3. MCMC provides an efficient means of mapping out the likelihood function over the full multi-dimensional parameter space and has a number of advantages over traditional maximum likelihood techniques.

For a particular choice of model parameters, one can construct a likelihood function which quantifies the agreement between the model and the data. Maximization techniques, such as the simplex algorithm, allow one to hone in on the "best-fit" model. However, with a large number of parameters, the likelihood function may become difficult to characterize with many false maxima. Moreover, the computational costs of simple algorithms, such as grid-based searches, become prohibitive.

The goal of our MCMC analysis is to calculate the posterior probability density function, $p(M \mid D, I)$, of a Galactic model, $M$, given data, $D$, and prior information, $I$. From Bayes' 
theorem we have

$$
p(M \mid D, I)=\frac{p(M \mid I) p(D \mid M, I)}{p(D \mid I)}
$$

where $p(M \mid I)$ is the prior probability density and $p(D \mid I) \equiv \int d \mathbf{A} p(M \mid D, I)$ is a normalization factor. In MCMC, one constructs a sequence or chain of models through parameter space chosen according to a prescribed algorithm. The distribution of models along the chain will be proportional to $p(M \mid D, I)$ provided the chain is sufficiently long.

In this work, we use the Metropolis-Hastings algorithm (Metropolis et al. 1953; Hastings 1970) as outlined in Gregory (2005). The first model in the chain is chosen at random. A candidate for the second model is chosen by taking a step in parameter space according to a proposal distribution. Let $\mathcal{R}$ be the ratio of the likelihood function of the candidate to that of the first model. The candidate is accepted a fraction, $f$, of the time where $f=\min \{1, \mathcal{R}\}$. Otherwise, the second model is taken to be identical to the first model. The process is repeated to find the third model and so forth.

Care must be taken in selecting a proposal distribution. If the step size is too short, the chain moves slowly through parameter space and the time required to fully explore parameter space becomes prohibitively large. On the other hand, if the typical step size is too large, the acceptance rate will be very low. We use an iterative approach, as outlined in Widrow, Pym, \& Dubinski (2007), to choose an efficient proposal distribution.

Since the data used in this work do not include observations of the velocity dispersion, the parameters $\sigma_{R 0}$ and $R_{\sigma}$ are superfluous and may be ignored in fitting the galaxy. Likewise, only the major-axis surface brightness profile is used and therefore the disk scale-height parameter, $z_{d}$, may be fixed to a reasonable value. Finally, $r_{h}$ may be set to any value greater than $15 \mathrm{kpc}$ (i.e., beyond the outermost point of the observed rotation curve) and $\delta r_{h}$ may be ignored. The DF is thus specified by ten free parameters.

Our set of model parameters must include the mass-to-light ratios of the disk and bulge. In general, the rotation curve fit for LSBs is improved by choosing a very large mass-to-light ratio for the disk, that is, by devising a galactic model that is disk-dominated in the inner regions. However, the required mass-to-light ratios are typically unphysical. Indeed, one can constrain mass-to-light ratios using population synthesis models and galaxy colours (Bell \& de Jong 2001; Bell et al. 2003). In a Bayesian analysis such as MCMC, these constraints are implemented through prior probabilities for the mass-to-light ratios. For simplicity, we assume that these prior probabilities follow a log-normal distribution. Using the $B-R$ and $B-V$ profiles from de Blok, van der Hulst, \& Bothun (1995) and the color-M/L relations from Bell et al. (2003) we find

$$
\log \left((M / L)_{\text {disk }}\right)=0.03 \pm 0.25
$$


and

$$
\log \left((M / L)_{\text {bulge }}\right)=0.2 \pm 0.25
$$

The errors, which translate directly into the width of the prior probability distribution, are meant to incorporate uncertainties in the relations from Bell et al. (2003), uncertainties in the colors, and differences in the $M / L$-values obtained by using either $B-R$ or $B-V$ colours.

Two MCMC runs are conducted, one in which $\gamma$ is a free parameter and one in which $\gamma$ is fixed to the NFW-value (i.e., $\gamma=1$ ). The surface brightness profile and rotation curve fits for a typical model from the first run are shown in Figure 4. Also shown is the fit found by McGaugh, Rubin, \& de Blok (2001) which assumes an exponential disk and does not include a bulge. Evidently, an excellent fit to the full surface brightness profile can be obtained provided both disk truncation and a bulge are included in the model. In Figure 5 we show the probability distribution function for $\gamma$. Clearly, the data favor values of $\gamma$ between 0 and 0.8 .

The surface brightness profile and circular speed curve for a typical model from our MCMC analysis with $\gamma=1$ is shown in Figure 6. We find that the model rotation curve rises too quickly as compared with the data, in agreement with previous studies (Moore 1994; Flores \& Primack 1994; McGaugh \& de Blok 1998; de Blok, McGaugh, \& Rubin 2001).

\section{MODELLING F-583 WITH A TRIAXIAL HALO}

Using the method outlined in Section 2, we transform the halo in one of our axisymmetric $\gamma=1$ models. We begin by generating an N-body representation of the model with $500 \mathrm{~K}$ particles for the halo, $400 K$ particles for the disk, and $100 K$ particles for the bulge. We produce two examples of models with triaxial halos: Model I where $\beta_{1}=0.24 \mathrm{Gyr}^{-1}$ and $\beta_{2}=0.08 \mathrm{Gyr}^{-1}$ (i.e., same choise of parameters as in our isolated halo example) and Model II where $\beta_{1}=0.16 \mathrm{Gyr}^{-1}$ and $\beta_{2}=-0.08 \mathrm{Gyr}^{-1}$. Note that the artificial acceleration is applied only to the halo particles.

First, consider Model I. Recall that in the example from Section 2 the short axis of the halo is aligned with the $z$-direction while the long axis is aligned with the $x$-direction. In a disk-bulge-halo system, the same choice of parameters leads to a rather mild deviation from axial symmetry since the intermediate axis is in the disk plane.

For an isolated halo, the choice of parameters used in Model II amounts to a trivial interchange of the $y$ and $z$ coordinates. In the presence of the disk, the choice yields a halo model in which both the long and short axes are in the disk plane and hence the departure 
from axial symmetry is very strong.

In Figure 7, we show contour plots of the disk surface density for Models I and II. As expected, departures from circular symmetry are more evident in Model II. Also, as expected, the long axis of the disk is perpendicular to the long axis of the halo Hayashi \& Navarro (2006).

In Figure 8 we show the evolution of the halo axes ratios as a function of time for Models I and II. The evolution of the axis ratios in Model I is very similar to that found for the isolated halo in Section 2. By contrast, the influence of the disk is readily evident in Model II; the halo is flattened along the $z$-direction and somewhat rounder in the $x-y$ plane than it would be in the absence of the disk. The end result is a halo that is prolate with approximate axial symmetry about the $x$-axis.

In Figure 9 we show the rotation curves for Models I and II as calculated along a slit placed on the major axis of the disk. In both experiments, the maximum rotation speeds decreases relative to their initial values by about $10 \mathrm{~km} \mathrm{~s}^{-1}$. One might imagine an iterative procedure in which, given these results, one adjusts the initial model so that the final system better reproduces the data.

Next, we consider the change in shape of the rotation curve produced by the deformation of the halo. In both experiments, the rotation curve rises more slowly than in the initial, axisymmetric model. Figure 9] shows the logarithmic slope of the rotation curve and illustrates this point quantitatively. We can also quantify the change in shape of the rotation curve by considering the fitting formula

$$
v(r)=v_{0} \frac{1}{\left(1+x^{\alpha}\right)^{1 / \alpha}}
$$

where $x=a_{v} / r$ Courteau (1997). $a_{v}$ and $v_{0}$ are scale parameters while $\alpha$ dictates the shape of the function. (Courteau (1997) actually proposed a more elaborate fitting formula but for our purposes, this form will suffice (see, for example, (Havashi et al. 2004).)) A coredisothermal sphere yields a rotation curve with $\alpha \geq 2$ while an NFW-halo yields a rotation curve with $\alpha \simeq 0.6$ (Courteau 1997; Havashi et al. 2004). We find the following values for $\alpha$ : observed rotation curve -5.35 ; initial, axisymmetric model - 0.91; Model I - 1.94; and Model II - 3.57. Clearly, Model II, where departures from axial symmetry are strongest, comes closest to reproducing the shape of the rotation curve. 


\section{SUMMARY AND CONCLUSION}

The adiabatic squeezing method Holley-Bockelmann et al. (2001) produces triaxial halos that have shrunk in size and therefore requires that the positions and velocities of the particles be rescaled. This awkward step precludes the technique from being applied to compound systems. Our approach avoids this problem by using an energy-conserving artificial force to deform the halos.

Our analysis of the LSB galaxy F568-3 begins with a discussion of axisymmetric models. We attempt to fit both photometric and kinematic observations using Bayesian statistics and the MCMC method. Our excellent fit of the surface brightness profile requires a bulge and disk truncation, neither of which were included in previous studies. As for the rotation curve, we find that constant density cores do better than density cusps in agreement with earlier studies of LSBs.

The second stage of our analysis is to deform the halo of a compound system. In agreement with Hayashi \& Navarro (2006), we show that the rotation curve of F568-3 may indicate the presence of a triaxial halo rather than a problem with the standard CDM model of structure formation. Hayashi \& Navarro (2006) and Hayashi, Navarro, \& Springel (2007) construct rotation curves by finding closed orbits in the gravitational potential of a triaxial halo. We calculate the rotation curves by making pseudo-observations of a disk that is self-consistently embedded in a dark halo.

There are two improvements that will add a further level of realism to the analysis: the inclusion of a gas disk in the galactic models and an iterative scheme whereby the initial model and artificial acceleration parameters are adjusted so that the final model fits the data in detail. These improvements will be considered in a future publication.

Our triaxial models have a wide range of applications. For example, they can be used to study the effect a non-spherical halo has on the morphology of tidal streams from satellite galaxies and flaring and warping of the gas disk. The method can also be applied to bulges where departures from axial symmetry are thought to be important.

It is a pleasure to thank J. Bailin, S. Courteau, J. Dubinski, S. McGaugh, and D. Puglielli for useful conversations. This work was supported by a grant from the Natural Sciences and Engineering Research Council of Canada. 


\section{REFERENCES}

Bailin, J. et al. 2007, ApJ, 667, 191

Bell, E. F. \& de Jong, R. S. 2001, ApJ, 550, 212

Bell, E. F. et al. 2003, ApJS, 149, 289

Binney, J., \& Tremaine, S. 1987, Galactic Dynamics, Princeton Univ. Press, Princeton

Bullock, J. S. et al. 2001, ApJ, 555, 240

Courteau, S. 1997, AJ, 114, 2402

de Blok, W. J. G., van der Hulst, J. M., \& Bothun, G. D. 1995, MNRAS, 274, 235

de Blok, W. J. G. \& McGaugh, S. S. 1998, ApJ, 508, 132

de Blok, W. J. G., McGaugh, S. S., \& Rubin, V. C. 2001, AJ, 122, 2381

Dehnen, W. 2001, ApJ, 536, L39

Dubinski, J. \& Carlberg, R. G. 1991, ApJ, 378, 496

Firmani, C. et al. 2000, MNRAS, 315, L29

Flores, R. A. \& Primack, J. R. 1994, ApJ, 427, L1

Freeman, K. C. 1970, ApJ, 160, 811 The

Gelman, A., Carlin, J. B., Stern, H. S., \& Rubin, D. B. 1995, Bayesian Data Analysis, Chapman \& Hall, London

Gregory, P. 2005, Bayesian Logical Data Analysis for the Physical Sciences, Cambridge University Press, UK

Hansen, S. H. \& Moore, B. 2006, New Astronomy, 11, 333

Hastings, W. K. 1970, Biometrika, 57, 97

Hayashi, E. et al. 2004, MNRAS, 355, 794

Hayashi, E. \& Navarro, J. F. 2006, MNRAS, 373, 1117

Hayashi, E., Navarro, J. F., Springel, V. 2007, MNRAS, 377, 50

Hoekstra, H., Yee, H.K.C., \& Gladders, M. D. 2004, ApJ, 606, 67 
Holley-Bockelmann, K. et al. 2001, ApJ, 549, 862

Johnston, et al. 1999, AJ, 118, 1719

Kuijken, K., \& Dubinski, J. 1995, MNRAS, 277, 1341

Mandelbaum, R. et al. 2006, MNRAS, 370, 1008

McGaugh, S. S. \& de Blok, W. J. G. 1998, ApJ, 499, 41

McGaugh, S. S., Rubin, V. C., \& de Blok, W. J. G. 2001, AJ, 122, 2396

Metropolis, N. et al. 1953, Journal of Chemical Physics, 21, 1087

Mo, H. J. \& Mao, S. 2000, MNRAS, 318, 163

Moore, B. 1994, Nature, 370, 629

Moore, B. et al, 2004, MNRAS, 354, 522

Navarro, J. F., Frenk, C. S., \& White, S. D. M. 1996, ApJ, 462, 563

Novak, G. S. et al. 2006, ApJ, 646, L9

Olling, R. P. \& Merrifield, M. R. 2000, MNRAS, 311, 361

Parker, L. C. et al. 2007, ApJ, 669, 21

Prugniel, P. \& Simien, F. 1997, A\& A, 321, 111

Stiff, D. 2003, PhD Thesis, Queen's University

Taylor, J. E. \& Navarro, J. F. 2001, ApJ, 563, 483

Terzić, B. \& Graham, A. W. 2005, MNRAS, 362, 197

Warren, M. S. et al. 1992, ApJ, 399, 405

Widrow, L. M. \& Dubinski, J. 2005, ApJ, 631, 838

Widrow, L. M., Pym, B, \& Dubinski, J. 2007, arXiv:0801:3414 


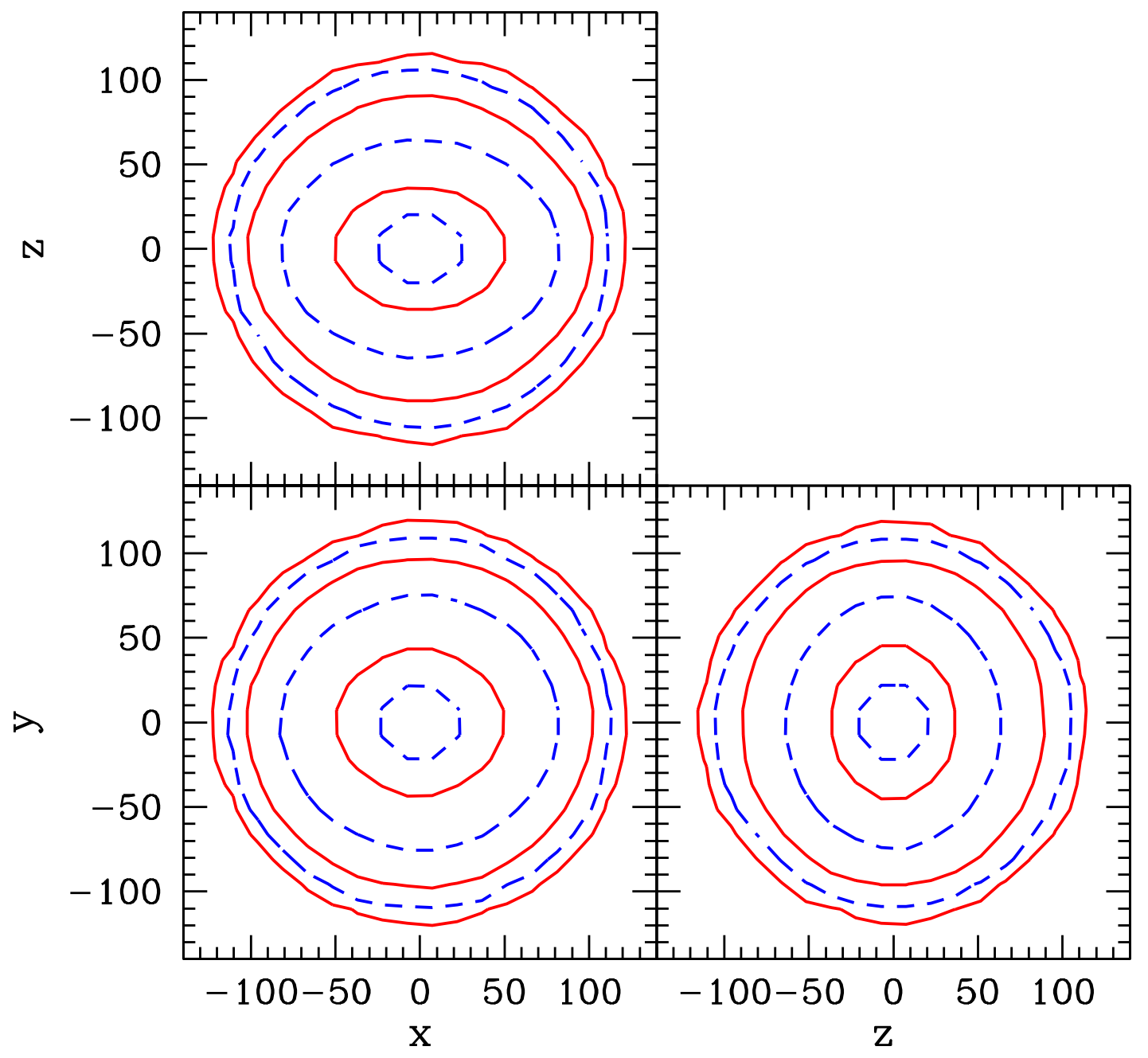

Fig. 1.- Surface density contours along the three principle axes for the triaxial isolated halo constructed in Section 2. Spacing between solid contours is 1 dex. 


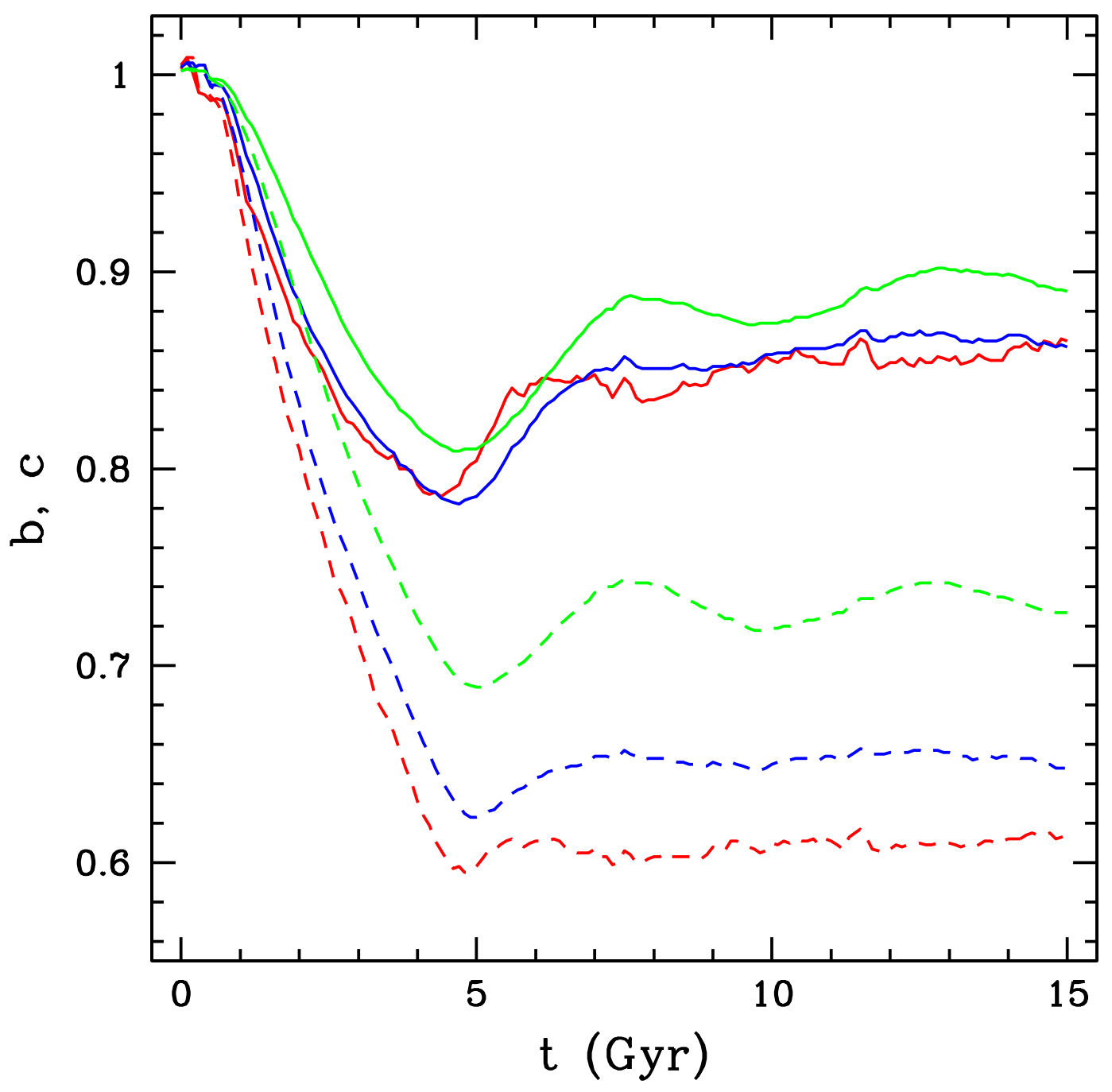

Fig. 2.- Axes ratios as a function of time. Solid curves show $b$; dashed curves show $c$. Red, blue, and green curves are for, respectively, the inner one third of the particles, the inner two thirds of the particles, and the entire halo. 


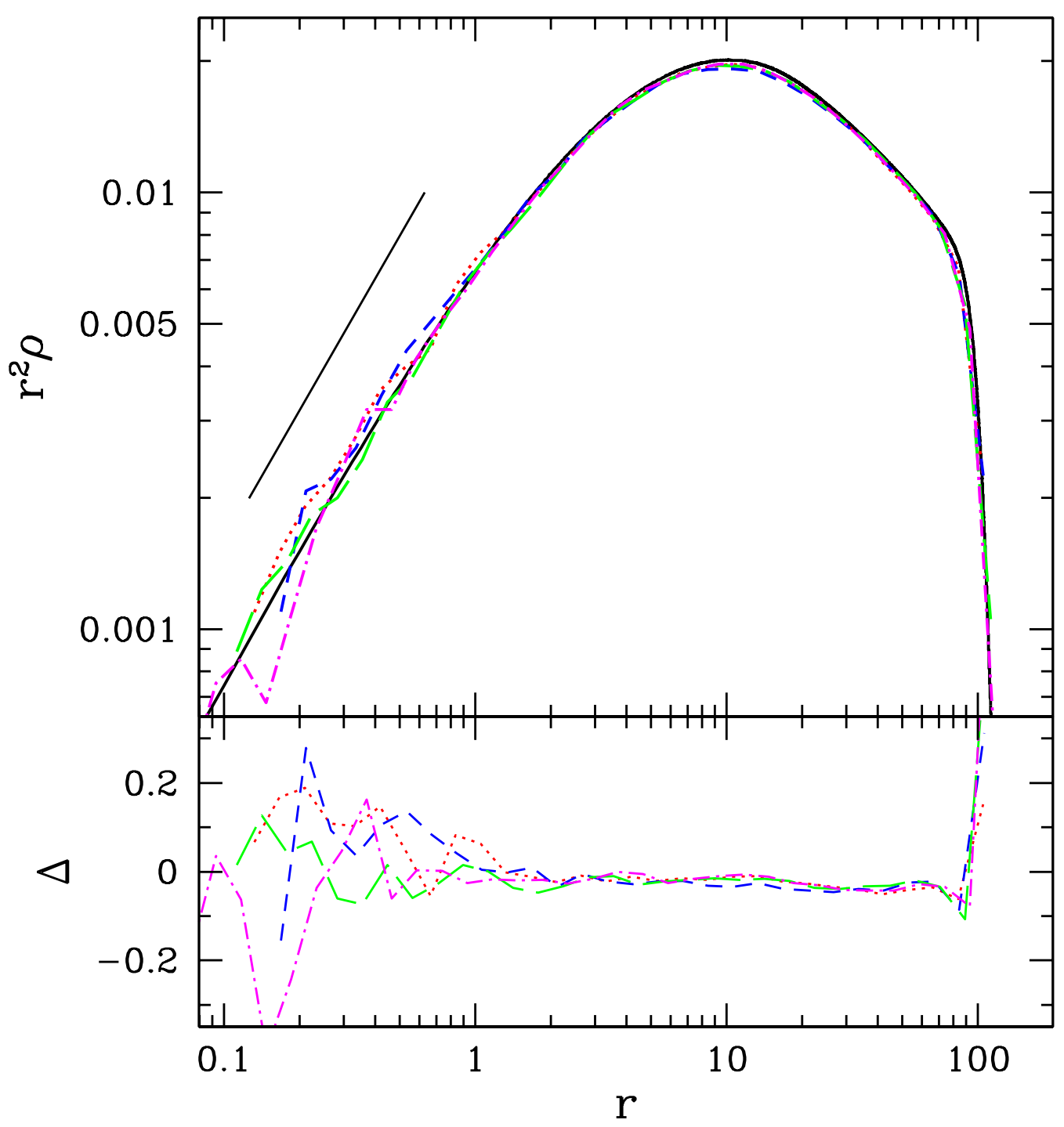

Fig. 3.- Differential mass profile, $d M / d r \propto r^{2} \rho$ as a function of spherical radius $r$. Line types are: analytic profile - solid black curve; initial profile derived from the N-body distribution - dot-dashed magenta curve; profile at $6 \mathrm{Gyr}$ - dotted red curve; profile at $12 \mathrm{Gyr}$ - dashed blue curve. The profile for the control experiment (no artificial acceleration) at $12 \mathrm{Gyr}$ is shown by the long-dashed green curve. The straight solid black line corresponds to $r^{2} \rho \propto r$ or $\rho(r) \propto r^{-1}$. Lower panel gives the fractional difference between the 4 measured profiles and the the analytic expression (i.e., $\left(\rho_{\text {measured }}-\rho_{\text {exact }}\right) / \rho_{\text {exact }}$. 
Table 1. Invariances of Equations 1-3

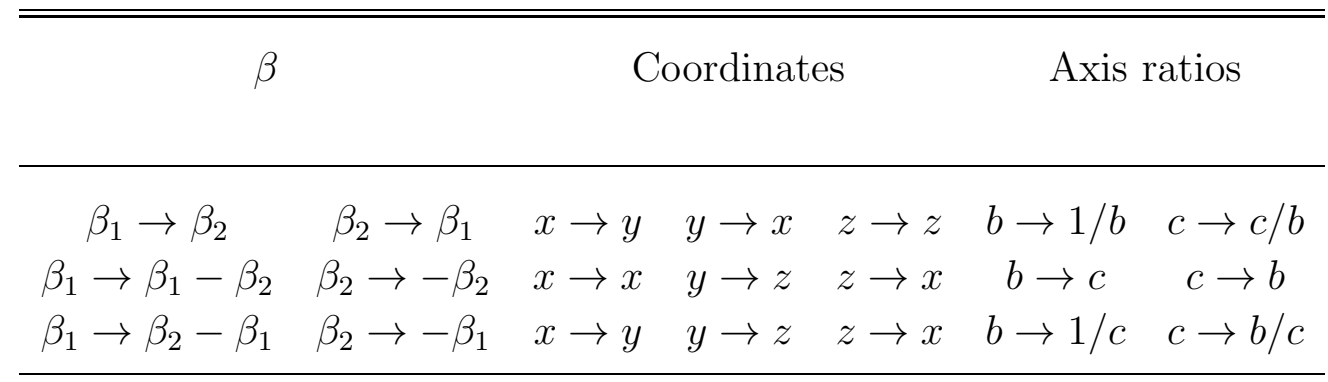

Table 2. Axis Ratios for Various Choices of $\beta_{1}$ and $\beta_{2}$

\begin{tabular}{|c|c|c|c|c|c|c|}
\hline \multirow[b]{2}{*}{ Model } & \multirow[b]{2}{*}{$\beta_{1}\left(\mathrm{Gyr}^{-1}\right)$} & \multirow[b]{2}{*}{$\beta_{2}\left(\mathrm{Gyr}^{-1}\right)$} & \multicolumn{2}{|c|}{ Isolated Halo } & \multicolumn{2}{|c|}{ Composite Model } \\
\hline & & & $\mathrm{b}$ & $\mathrm{c}$ & $\mathrm{b}$ & $\mathrm{c}$ \\
\hline $1 \mathrm{a}$ & 0.12 & 0.04 & 0.93 & 0.86 & 0.95 & 0.85 \\
\hline $1 b$ & 0.08 & -0.04 & 0.86 & 0.93 & 0.88 & 0.90 \\
\hline $1 \mathrm{c}$ & -0.08 & -0.12 & 0.93 & 1.07 & 0.93 & 1.06 \\
\hline $2 \mathrm{a}$ & 0.16 & 0.16 & 1.00 & 0.78 & 1.01 & 0.77 \\
\hline $2 \mathrm{~b}$ & 0.0 & -0.16 & 0.78 & 1.00 & 0.80 & 0.99 \\
\hline $3 a$ & 0.24 & 0.08 & 0.89 & 0.73 & 0.92 & 0.73 \\
\hline $3 \mathrm{~b}$ & 0.16 & -0.08 & 0.73 & 0.89 & 0.75 & 0.87 \\
\hline $3 c$ & -0.16 & -0.24 & 0.82 & 1.12 & 0.82 & 1.08 \\
\hline
\end{tabular}




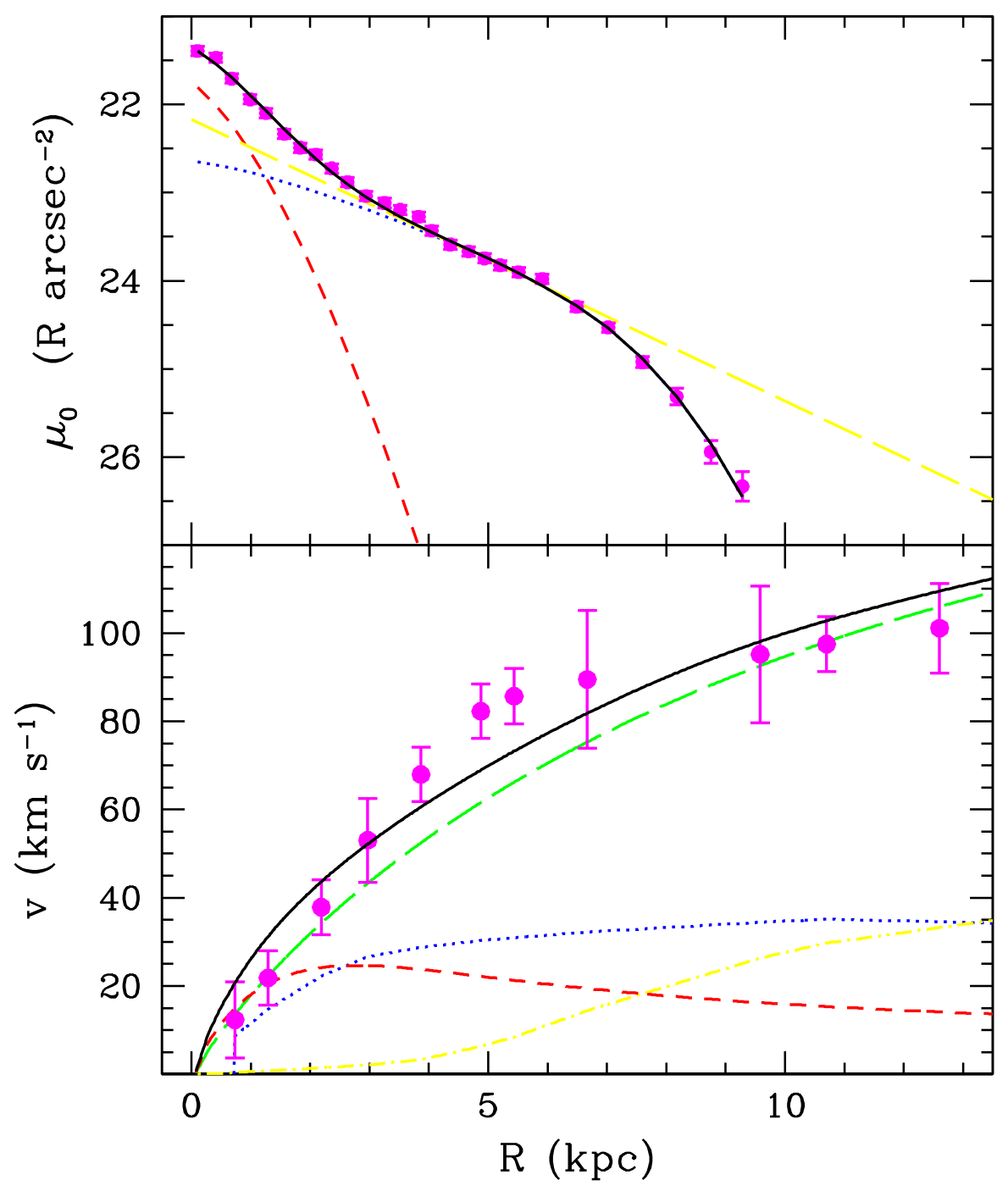

Fig. 4.- Comparison of data with predictions for a typical model from the MCMC run where $\gamma$ is a free parameter. Top panel shows surface brightness profile. Observations from de Blok, van der Hulst, \& Bothun (1995) are indicated by magneta dots. Line types are as follows: total model surface brightness profile - solid black curve; disk contribution - blue dotted curve; bulge contribution - red dashed curve; exponential disk model from de Blok, van der Hulst, \& Bothun (1995) - yellow long-dashed curve. Bottom panel shows the rotation curve. Observations from McGaugh, Rubin. \& de Blok (2001) are indicated by magneta dots. Total model - solid black curve; disk contribution - blue dotted curve; bulge contribution - red dashed curve; halo contribution - green long-dashed curve; gas contribution - yellow dot-dashed curve. 


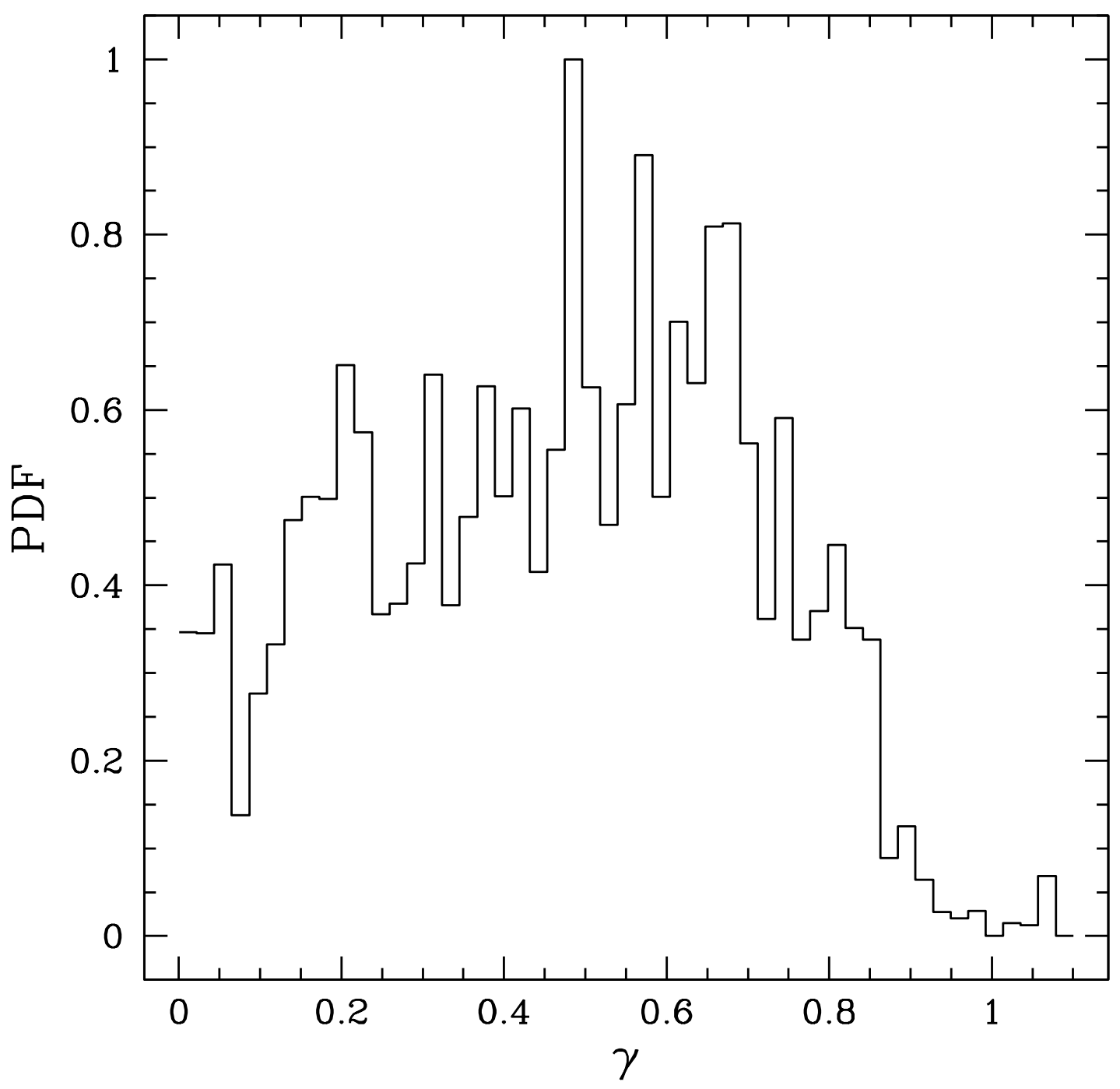

Fig. 5.- Probability distribution function for $\gamma$ from the first MCMC where $\gamma$ is a free parameter. 


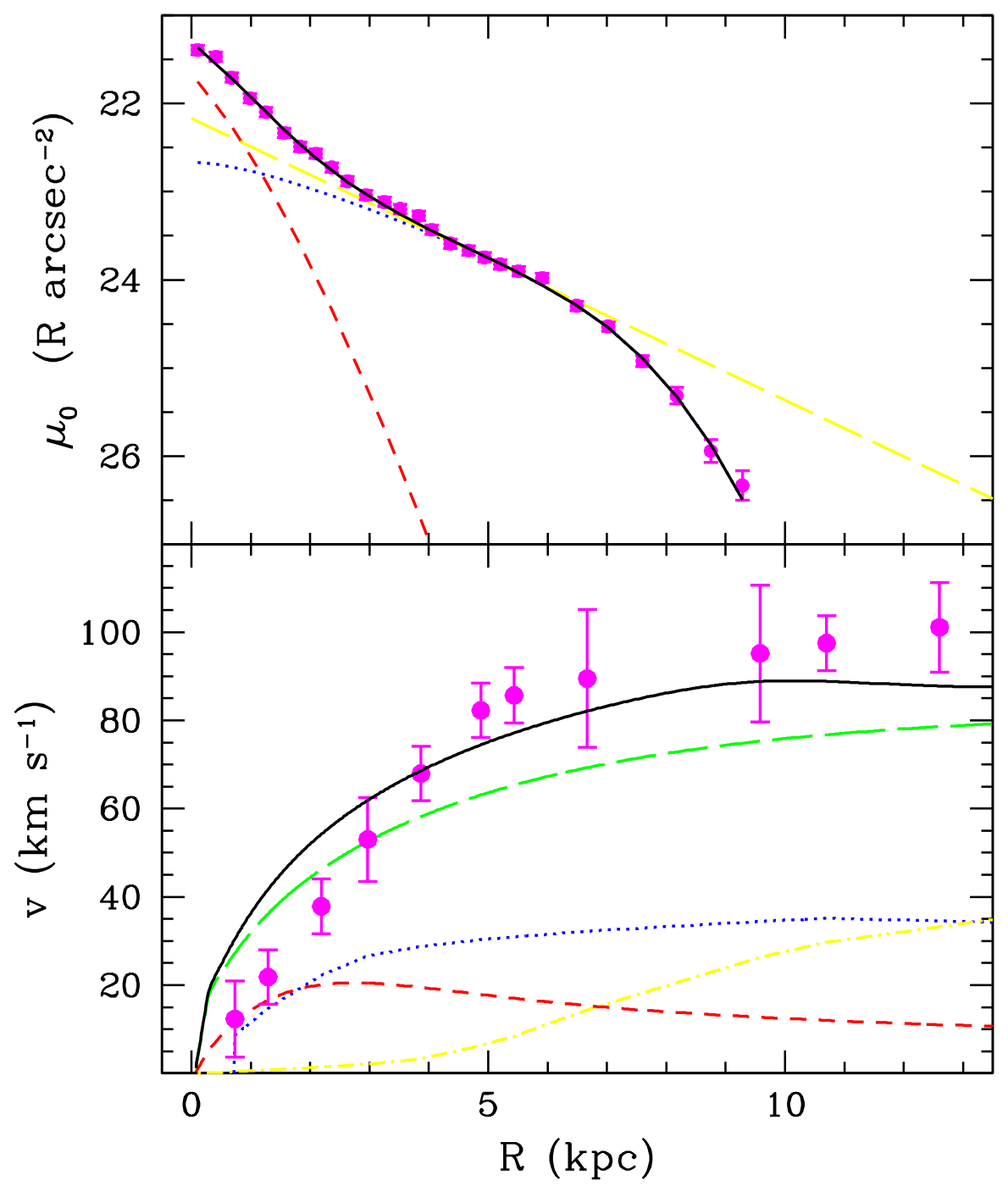

Fig. 6. - Surface brightness profile and circular speed curve for a typical model from MCMC chain where $\gamma=1$. Line types are the same as in Figure 4. 


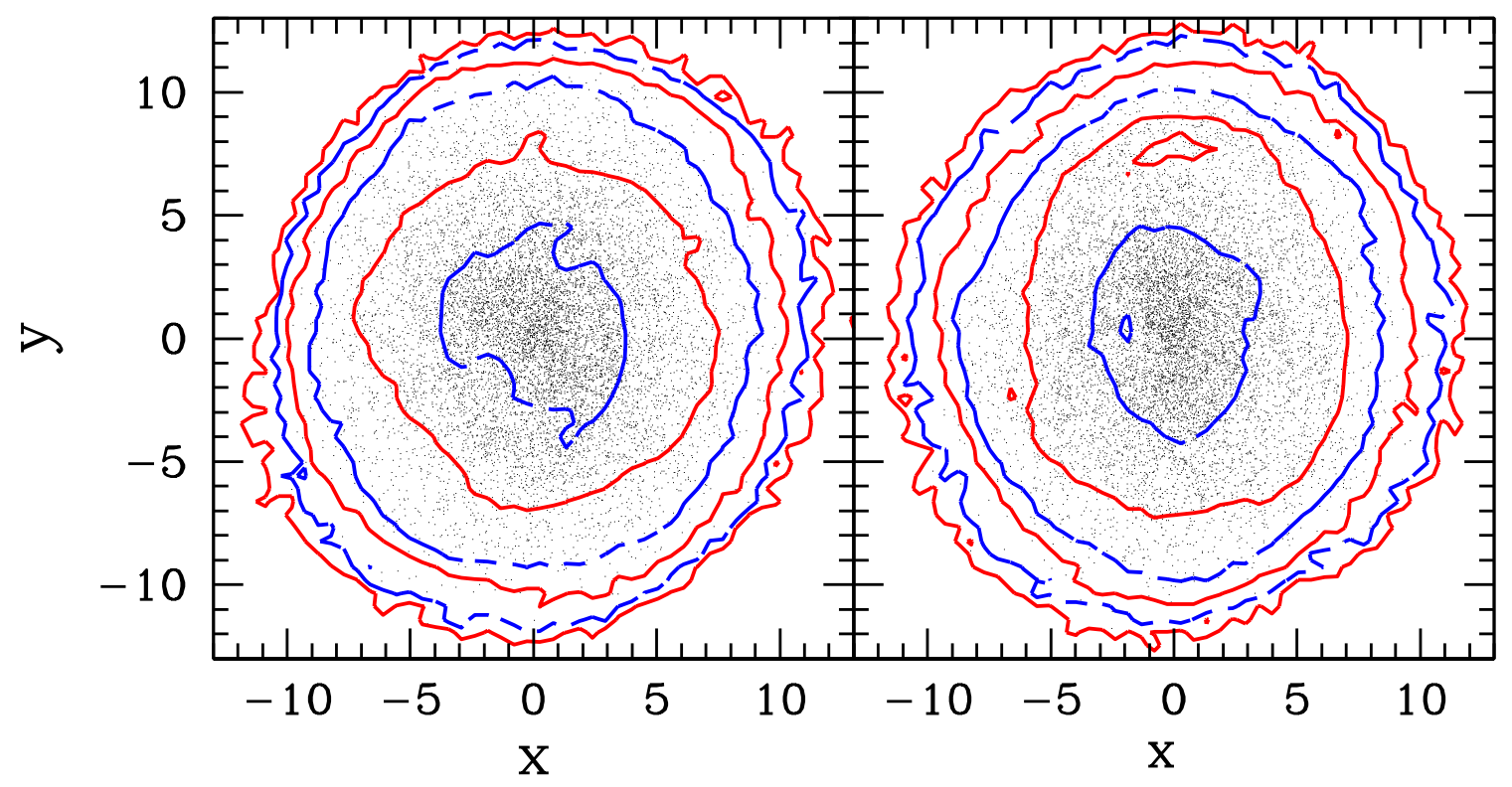

Fig. 7.- Superposition of surface density contours and projected particle distribution for Models A (top) and B (bottom). 


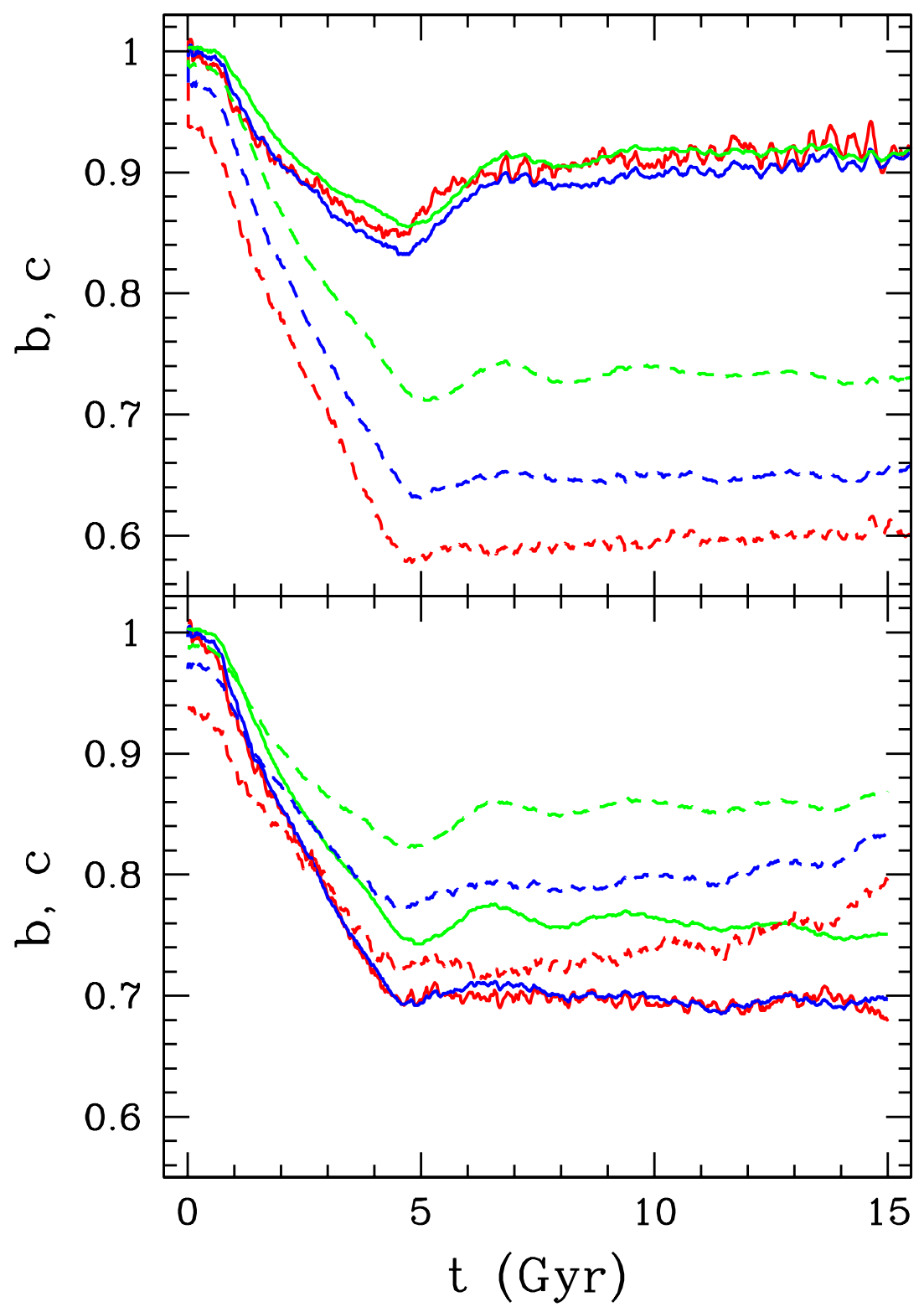

Fig. 8.- Halo axis ratios as a function of time for Models A (top) and B (bottom). Line types are the same as in Figures 2. 


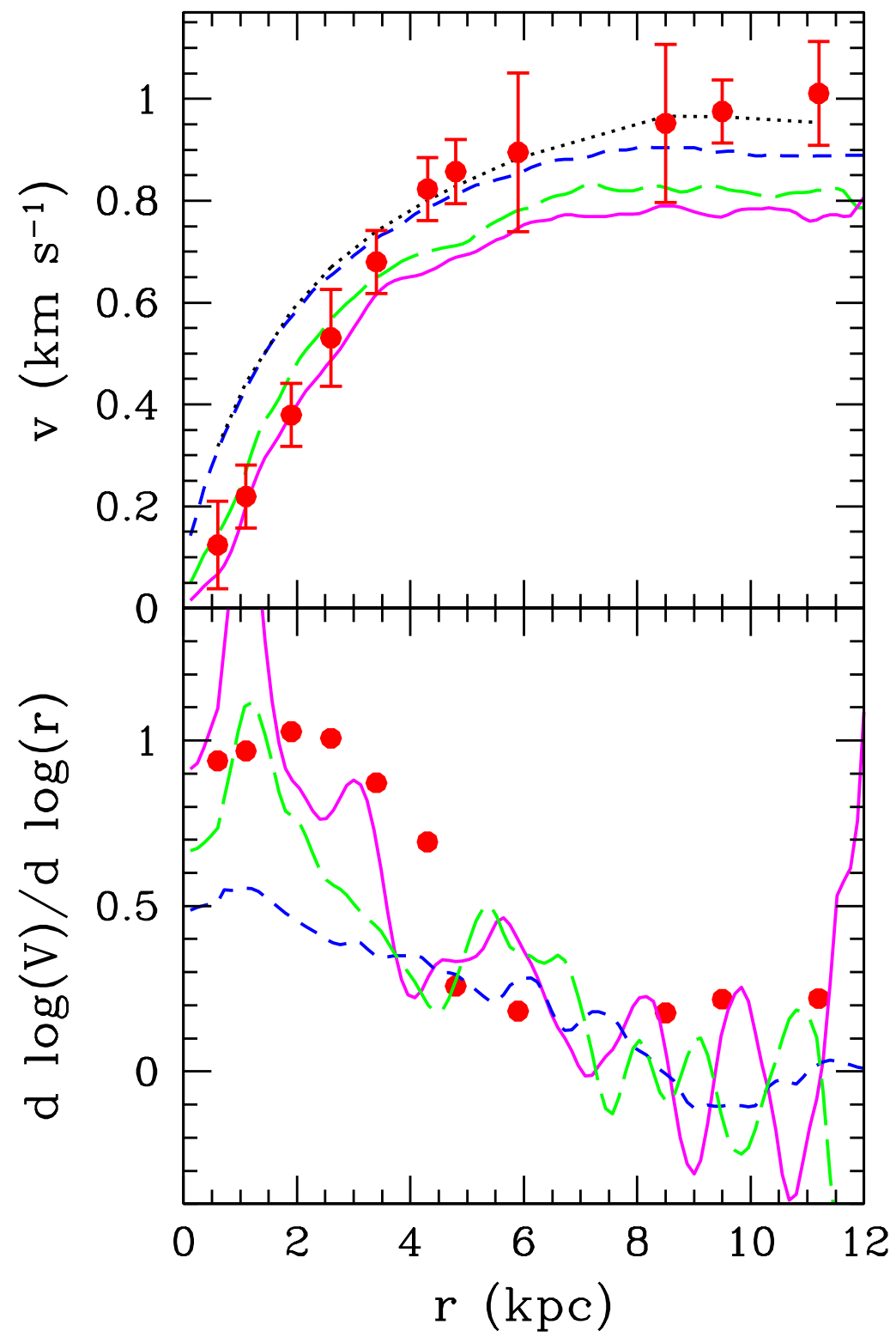

Fig. 9.- Observed and model rotation curves. Top panel shows the rotation curves; bottom panel shows the logarithmic slope. Observations from McGaugh, Rubin, \& de Blok (2001) are shown as red dots. Line types are as follows: black dotted line - rotation curve for the initial model as derived from the potential; blue dashed line - rotation for the initial model as derived by measuring bulk motion of the disk stars; green long-dashed line - rotation curve for Model I; magenta solid line - rotation curve for Model II. 